\title{
ESPACIOS DE PROBABILIDADES INDUCIDOS \\ POR FUNCIONES
}

ENRIQUE RAMOS MEZA. (*)

RESUMEN.

Se estudia el traslado de un espacio de proba bilidades, en el sentido de Kolmogorof, a un conjunto cualquiera a través de una función $f$. El conjunto pue de ser el espacio de partida o llegada de dicha función generando así los espacios de probabilidad "Inicial" y "Final" respectivamente, inducidos por $f$. Generar el es pacio de probabilidad final no constituye problema algu no, no ocurriendo igual cosa con la situación dual, en donde para trasladar la función de probabilidad hasta el conjunto (espacio de partida de f) se requiere del. con cepto de función "casi epiyectiva", con lo cual se solü ciona el problema.

ESPACIOS DE PROBABILIDADES DE KOLMOGOROF.

Se entiende por un espacio de probabilidades de Kolmogorof, un trío de la forma $(\Omega, \mathcal{F}, \mathbb{P})$; en don de $\Omega$ es un conjunto no vacío llamado "Espacio Muestral", $F$ una $\sigma$-álgebra de conjuntos para $\Omega$. 0 sea, se cum ple :

(*) Master en Estadística Matemática.

Profesor Depto.Matemáticas U. del Norte 
(a) $\Phi \varepsilon F$

(b) Si $A \in F$, entonces $A^{C} \varepsilon F$,

(c) para $\left\{A_{n} / n \in \mathbb{N}\right\} \leq F$, se tiene:

$$
\underset{n \varepsilon \mathbb{N}}{U} A_{n} \varepsilon F
$$

Los elementos de $F$ son interpretados como "su cesos". Finalmente $\mathbb{P}$ es una función real valorada sobre $F$, llamada "función de probabilidad" que satisface las tres condiciones siguientes:

(a) $\mathbb{P}(\mathrm{A}) \geqslant 0, \forall A \in F$

(b) para toda familia disjunta

$$
\begin{aligned}
& \left\{A_{n} / n \in \mathbb{N}\right\} \subseteq F \\
& \text { se tiene: } \\
& \mathbb{P}\left(\underset{n \in \mathbb{N}}{U} A_{n}\right)=\sum_{n \in \mathbb{N}} \mathbb{P}\left(A_{n}\right)
\end{aligned}
$$

(c) $\mathbb{P}(\Omega)=1$

O sea, se trata de una medida finita y normalizada sobre el espacio medible $(\Omega, F)$.

\section{ESPACIO DE PROBABILIDAD FINAL.}

Sea $(\Omega, F, \mathbb{P})$ un Espacio de probabilidades en el sentido de Kolmogorof. Si $\mathbb{E}$ es cualquier conjun to y $f=\Omega \rightarrow \mathbb{E}$ una función cualesquiera, la estructu- 
$\mathrm{ra}(\Omega, F, \mathbb{P})$ puede ser trasladada hacie $\mathbb{E}$ median te f. Para esto basta considerar una $\sigma$-álgebra sobre $\mathbb{E}$, que haga de $f$ una función medible, y una medida de probabilidad definida sobre el espacio medible formado. La $\sigma$-álgebra se llama " $\sigma$-álgebra final" y la probabiIidad "Probabilidad Final"; inducida por f respectiva mente.

CONSTRUCCION DE LA $\sigma$-ALGEBRA FINAL.

Se define la siguiente colección de conjuntos:

$$
F^{f}=\left\{\varepsilon \subseteq \mathbb{E} / f^{-1}(\varepsilon) \varepsilon F\right\}
$$

que cumple (a), (b), y (c) de (1); o sea, $F^{f}$ es, una $\sigma$-álgebra de conjuntos para $\mathbb{E}$. F es llamada " $\sigma$-ál gebra Final" inducida por $f$; y es la mayor $\sigma$-álgebra que hace de $f$ una función medible.

Se demuestra a continuación que $F^{f}$, es, efectivamente una $\sigma$-álgebra para $\mathbb{E}$.

(a) Como $f^{-1}(\mathbb{E})=\Omega$, y $\Omega c=\Phi \varepsilon F$ se tie ne que:

$$
\begin{aligned}
& {\left[f^{-1}(\mathbb{E})\right]^{c}=f^{-1}(\Phi) \varepsilon F \text {. Es decir }} \\
& \Phi \varepsilon \mathcal{F}^{f} .
\end{aligned}
$$

(b) Si $\varepsilon \varepsilon \mathcal{F}^{f}, f^{-1}(\varepsilon) \varepsilon F$. Como $F$ es

$$
\begin{aligned}
& \sigma \text {-álgebra, se tiene: }\left[f^{-1}(\varepsilon)\right]^{\subset} \varepsilon F \text {. } \\
& 0 \text { sea, } \varepsilon^{\propto} \varepsilon F \text {. }
\end{aligned}
$$

(c) Sea $\left\{A_{n} / n \in \mathbb{N}\right\} \subseteq F^{f}$, es decir 


$$
f^{-1}\left(A_{n}\right) \varepsilon \quad F^{f}, \forall n \in \mathbb{N}
$$

Nuevamente, como $F$ es $\sigma$-álgebra resulta:

$$
\underset{n \in \mathbb{N}}{\bigcup} f^{-1}\left(A_{n}\right) \varepsilon F \text {, de donde: }
$$

$$
\underset{n \in \mathbb{N}}{U} A_{n} \varepsilon F^{f}
$$

La maximilidad de $\mathcal{F}^{\mathrm{f}}$ es clara. Basta conside rar la función medible $f=(\Omega, F)+\left(\Omega *, F * F^{*}\right)$. Entonces para todo conjunto en $\mathcal{F}^{*}$, digamos $A$, se tiene que $\mathrm{f}^{-1}(\mathrm{~A}) \cdot \varepsilon \mathcal{F}$, luego, $A \varepsilon F f^{\circ}$. 0 sea, $F_{*} \subseteq F f$.

CONSTROCCION DE LA FUNCION DE PROBABILIDAD FINAL.

Defínase la siguiente medida sobre $\left(\mathbb{E}, F^{f}\right)$.

$$
\mathbb{P}^{f}(\varepsilon)=\mathbb{P}\left[f^{-1}(\varepsilon)\right], V \varepsilon \varepsilon F^{f}
$$

que cumple (a), (b) y (c) de (2). O sea, es una medida de probabilidad finita y normalizada sobre el espaciome dible ( $\mathbb{E}, \mathcal{F} f$ ) llamada "Función de probabilidad Final" inducida por $f$.

Se demuestra ahora que efectivamente $\mathbb{P}^{\mathrm{f}}$ es una medida de probabilidad.
(a) Como $\mathbb{P}\left[\mathrm{f}^{-1}\right.$
$(\varepsilon)]>0, \mathrm{Vf}^{-1}$
(E) $\varepsilon F$,
es inmediato que $\mathbb{P}^{f}(\varepsilon) \geqslant 0, \forall \varepsilon \varepsilon F^{f}$. 
(b) Sea la colección disjunta $\left\{A_{n} / n \in \mathbb{N}\right\} \subseteq F^{f}$, entonces:

$$
\begin{aligned}
\mathbb{P}^{f}\left(\underset{n \in \mathbb{N}}{U} A_{n}\right) & =\mathbb{P}\left[f^{-1}\left(\underset{n \in \mathbb{N}}{U} A_{n}\right)\right] \\
& =\mathbb{P}\left[\underset{n \in \mathbb{N}}{U} f^{-1}\left(A_{n}\right)\right] \\
& =\sum_{n \in \mathbb{N}} \mathbb{P}\left[f^{-1}\left(A_{n}\right)\right] \\
& =\sum_{n \in \mathbb{N}} \mathbb{P}^{f}\left(A_{n}\right)
\end{aligned}
$$

(c) Puesto que $f^{-1}(\mathbb{E})=\Omega$, es claro que $\mathbb{P}^{f}(\mathbb{E})=1$

CONCLUSION.

Para $f:(\Omega, F, \mathbb{P})+\mathbb{E} ;\left(\mathbb{E}, F^{f}, \mathbb{P}^{f}\right)$ se llama "Espacio de probabilidad final" inducido por la función $f$. Dicho nombre se debe a que la estructura se forma en el espacio de llegada de $f$. De importancia re sulta el caso $\mathbb{E}=\mathbb{R}$ y $F f=B$ donde $B$ denota la $\sigma$-álgebra de conjuntos de Borel en $\mathbb{R}$; pues $f$, en es te caso, es llamada "Variable estocástica" y permite aplicar el cálculo matemático a la teoría de la Probabi lidad. De igual interés resultan los casos $\mathbb{E}=\mathbb{R}^{\mathrm{n}} \overline{\mathrm{y}}$ $E=\mathbb{C}$.

ESPACIO DE PROBABILIDAD INICIAL.

Se considera ahora la función $f: \mathbb{E} \rightarrow \Omega$. EI objetivo será trasladar la estructura $(\Omega, \dot{F}, \mathbb{P})$ median 
te f hasta $\mathbb{E}$. 0 sea, se trata del caso dual al presen tado anteriormente.

Resulta aquí, que la estructura $(\Omega, F, \mathbb{P})$ sólo puede ser trasladada parcialmente hasta $\mathbb{E}$. Obtener una $\sigma$-álgebra para $\mathbb{E}$ inducida por $f$ no presenta problemas, sin embargo, la media $\mathbb{P}$ no puede ser llevada a $\mathbb{E}$, a menos que se introduzca una condición adicio nal a $f$. Tal condición consiste en imponer que $f$ sea "casi-epiyectiva" ; tal como se indica en el comienzo de estas notas.

CONSTRUCCION DE LA $\sigma$-ALGEBRA. INICIAL.

Se define la siguiente colección de conjuntos:

$$
F_{\mathrm{f}}=\left\{\mathrm{f}^{-1}(\varepsilon) \leq \mathbb{E} / \varepsilon \varepsilon F\right\}
$$

que cumple (a), (b) y (c) de (1). O sea, $F$, es una $\sigma$-álgebra de conjuntos para $\mathbb{E}$. $F_{f}$ es llamada " $\sigma$-ál gebra Inicial" inducida por $f$ y es la menor $\sigma$-álgebra que hace de $f$ una función medible.

Se demuestra a continuación que $F_{f}$ es una $\sigma$-álgebra para $\mathbb{E}$.

(a) Ya que $\Omega^{c} \varepsilon F$, entonces $f^{-1}\left(\Omega^{*}\right) \varepsilon F_{f}$.

$$
\begin{aligned}
& \text { Pero como } f^{-1}(\Omega)=\mathbb{E} \text {, resulta } \\
& f^{-1}\left(\Omega^{c}\right)=\Phi \text {. } 0 \text { sea, } \Phi \varepsilon F_{f^{*}}
\end{aligned}
$$

(b) Si $A \in F_{f}$; existe $\varepsilon \in F$, tal que $\mathrm{f}^{-1}(\varepsilon)=$ A. Como $F$ es $\sigma$-álgebra, 


$$
\begin{aligned}
& \mathcal{E}^{c} \varepsilon F \text {. Por lo tanto, } f^{-1}\left(\varepsilon^{c}\right) \varepsilon F_{f}, \\
& \text { de donde }\left[f^{-1}(\varepsilon)\right]^{c} \varepsilon F_{f} \text {. O sea } \\
& A^{c} \varepsilon F_{f} \text {. }
\end{aligned}
$$

(c) Sea $\left\{A_{n} / n \varepsilon \mathbb{N}\right\}=F_{f} \cdot 0$ sea, existen

$\varepsilon_{n} \varepsilon F$ tal que $A_{n}=f^{-1}\left(\varepsilon_{n}\right), \forall n \in \mathbb{N}$.

Como $F$ es una $\sigma$-álgebra, resulta

$\underset{\mathrm{n} \in \mathbb{N}}{U} \varepsilon_{\mathrm{n}} \in F . \quad 0$ sea,

$$
\underset{n \in \mathbb{N}}{\cup} A_{n} \varepsilon F_{f}
$$

La minimalidad de $F_{f}$ es clara. Basta considerar la fun ción medible. $f:\left(\Omega^{*}, F^{*}\right) \rightarrow(\Omega, F)$, entonces para todo $A$, conjunto en $F$, se tiene $f^{-1}$ (A) $\varepsilon F^{*}$. De don$\operatorname{de} \bar{F}_{f}=F^{*}$.

Veamos ahora el problema de dotar al espacio medible $\left(\mathbb{E}, F_{f}\right)$ de una medida de probabilidad apropia $d a$, inducida por la función $f$. Para tal efecto tomamos $f$ eniyectiva, excepto en un conjunto de medida cero ( 0 sea, que exista $\varepsilon \varepsilon \bar{F}$ con $\mathbb{P}(\varepsilon)=0$, tal que $\left.f(\mathbb{E})^{c} \subseteq \mathcal{E}\right)$. Tales funciones son llamadas "funcio nes casi-epiyectivas".

Un resultado importante y de mucha utilidad en el logro de este objetivo es el siguiente: Si $\varepsilon$ es un conjunto en $F$ de medida cero, entonces para $\varepsilon_{1}$ y $\varepsilon_{2}$ en $F$ que cumplan $\mathrm{P}^{-1}\left(\varepsilon_{1}\right)=\mathrm{f}^{-1}\left(\varepsilon_{2}\right)$, se tiene $\varepsilon_{1} \Delta \varepsilon_{2}=\varepsilon$ 
donde $\Delta$ denota "diferencia simétrica". Esto conduce a que $\mathbb{P}\left(\varepsilon_{1} \Delta \varepsilon_{2}\right)=0$. 0 sea, $\mathbb{P}\left(\varepsilon_{1} \cup \varepsilon_{2}\right)=\mathbb{P}\left(\varepsilon_{1} \cap \varepsilon_{2}\right)$ obteniendo finalmente $\mathbb{P}\left(\varepsilon_{1}\right)=\mathbb{P}\left(\varepsilon_{2}\right)$. (*).

CONSTRUCCION DE LA FUNCIOH DE PROBABILIDAD INICIAL.

sobre $\mathbb{E}$ :

Se define la siguiente medida de probabilidad

$$
\mathbb{P}_{f}\left[f^{-1}(\varepsilon)\right]=\mathbb{P}(\varepsilon), \quad \forall \varepsilon \varepsilon F
$$

que gracias al resultado anterior cumple con (a), (b) y (c) de (2). O sea, es una medida de probabilidad finita y normalizada sobre el espacio medible $\left(\mathbb{E}, F_{f}\right)$ llamada "Función de probabilidad inicial" inducida por $f$.

Se demuestra a continuación que $\mathbb{P}_{f}$ efectivamente es una función de probabilidad:

(a) Para cualquier A $\varepsilon F_{f}$, existe $\varepsilon \varepsilon F$ tal que $A=f^{-1}(\varepsilon)$. Luego $\mathbb{P}_{f}(A)=\mathbb{P}(\varepsilon)$ y como $\mathbb{P}(\varepsilon) \geqslant 0$ resulta $\mathbb{P}_{f}(A) \geqslant 0$.

(b) Sea $\left\{A_{n} / n \in \mathbb{N}\right\} \leq F_{f}$ disjunta. 0 sea, existen $\varepsilon_{n} \varepsilon F$ tal que : $A_{n}=f^{-1}\left(\varepsilon_{n}\right), \forall n \varepsilon \mathbb{N}$. Obsérvese que $\varepsilon_{n} \cap \varepsilon_{\text {m }} \subseteq[f(\mathbb{E})]=$. Construyen do conjuntos $\varepsilon^{\prime}$, mediante: 


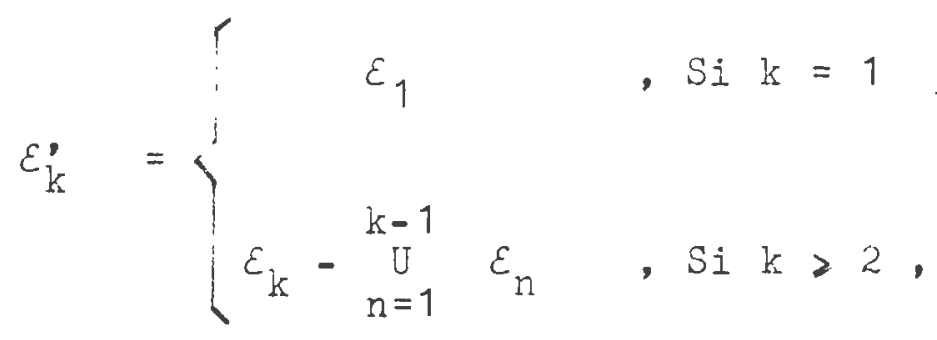

Se tiene $\left\{\varepsilon_{\mathrm{n}}^{\prime} / \mathrm{n} \varepsilon \mathbb{\mathbb { T }}\right\}$ disjuntos y $\underset{n \in \mathbb{I} !}{U} \varepsilon_{\mathrm{n}}^{\prime}=\underset{\mathrm{n} \varepsilon \mathbb{N}}{\mathbb{U}} \varepsilon_{\mathrm{n}}$.

Usando (*) resulta:

$$
\begin{aligned}
& \mathbb{P}_{f}\left(\underset{n \in \mathbb{N}}{U} A_{n}\right)=\mathbb{P}_{f}\left[\underset{n \in \mathbb{N}}{U} f^{-1}\left(\varepsilon_{n}^{\prime}\right)\right] \\
&=\mathbb{P}_{f}\left[f^{-1}\left(\underset{n \varepsilon \mathbb{N}}{U} \varepsilon_{n}^{\prime}\right)\right] \\
&=\mathbb{P}\left(\underset{n \in \mathbb{N}}{U} \varepsilon_{n}^{\prime}\right) \\
&=\sum_{n \in \mathbb{N}} \mathbb{P}_{n}\left(\varepsilon_{n}^{\prime}\right) \\
&=\sum_{n \in \mathbb{N}} \mathbb{P}_{f}\left(A_{n}\right) \\
& \text { (c) } \mathbb{P}_{f}(\mathbb{E})=\mathbb{P}_{f}\left[f^{-1}(\Omega)\right]=\mathbb{P}(\Omega)=1
\end{aligned}
$$

CONCLUSIOI.

Para $f: \mathbb{E}+(\Omega, F, \mathbb{P})$ la extructura

( $\mathbb{E}, F_{f}, \mathbb{P}_{f}$ ) se llama "Espacio de probabilidad inicial" inducido por fi, pues su formación se logra en el espacio de partida de f. Adjuntando a este resultado el ante rior, podemos decir que se ha cumplido el objetivo trazado. 


\section{BIBLIOGRAFIA.}

- J. GONZALEZ.: "Teoría de Probabilidades". U.T.E. Facultad de Ciencias Santiago. (1976).

- J. GOHZALEZ.: "Seminario de Procesos Estocásticos",J.T.E. Facultad de Ciencias, Santiago, (1977).

- J. MICHELOn.: "Topología". U.T.E. Facultad de Ciencias Santiago.

- D. MARTIigz.: "Seminario teoría de la medida". U. de Chile Sede Antofagasta, Departamento Mate máticas. (1980). 